\title{
Exploring the Usefulness of Finger-Based 3D Gesture Menu Selection
}

\author{
Arun Kulshreshth \\ Department of EECS \\ University of Central Florida \\ 4000 Central Florida Blvd \\ Orlando, FL 32816, USA \\ arunkul@knights.ucf.edu
}

\author{
Joseph J. LaViola Jr. \\ Department of EECS \\ University of Central Florida \\ 4000 Central Florida Blvd \\ Orlando FL 32816, USA \\ jj1@eecs.ucf.edu
}

\begin{abstract}
Counting using one's fingers is a potentially intuitive way to enumerate a list of items and lends itself naturally to gesturebased menu systems. In this paper, we present the results of the first comprehensive study on Finger-Count menus to investigate its usefulness as a viable option for 3D menu selection tasks. Our study compares 3D gesture-based finger counting (Finger Count menus) with two gesture-based menu selection techniques (Hand-n-Hold, Thumbs-Up), derived from existing motion-controlled video game menu selection strategies, as well as 3D Marking menus. We examined selection time, selection accuracy and user preference for all techniques. We also examined the impact of different spatial layouts for menu items and different menu depths. Our results indicate that Finger-Count menus are significantly faster than the other menu techniques we tested and are the most liked by participants. Additionally, we found that while Finger-Count menus and 3D Marking menus have similar selection accuracy, Finger-Count menus are almost twice as fast compared to 3D Marking menus.
\end{abstract}

\section{Author Keywords}

Menu Selection; Finger-Count menu; Hand-n-Hold Menu; Thumbs-Up Menu; 3D Marking Menu; Depth Camera; Gesture Recognition; Selection; 3D Interaction; User Study; Video Games.

\section{ACM Classification Keywords}

H.5.2 User Interfaces: Graphical user interfaces (GUI), input devices and strategies, interaction styles; H.5.m Information Interfaces and Presentation (e.g. HCI): Miscellaneous.

\section{General Terms}

Design, Experimentation, Measurement, Performance, Human Factors.

Permission to make digital or hard copies of all or part of this work for personal or classroom use is granted without fee provided that copies are not made or distributed for profit or commercial advantage and that copies bear this notice and the full citation on the first page. Copyrights for components of this work owned by others than ACM must be honored. Abstracting with credit is permitted. To copy otherwise, or republish, to post on servers or to redistribute to lists, requires prior specific permission and/or a fee. Request permissions from permissions@ acm.org.

CHI 2014, April 26-May 1, 2014, Toronto, Ontario, Canada.

Copyright (c) 2014 ACM 978-1-4503-2473-1/14/04..\$15.00.

http://dx.doi.org/10.1145/2556288.2557122

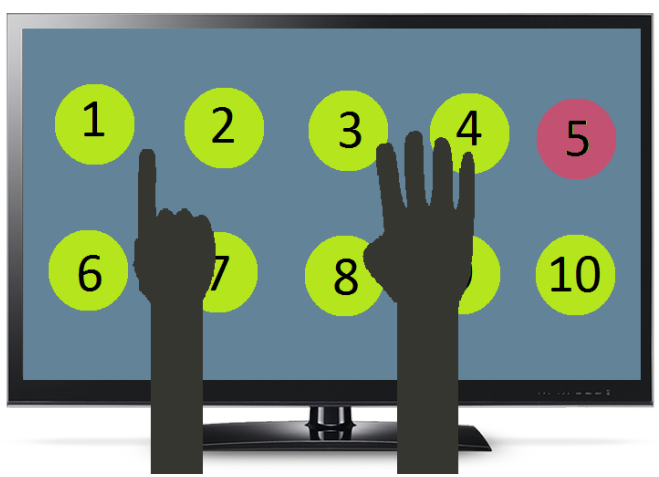

Figure 1. Finger-based menu selection

\section{INTRODUCTION}

Menu systems are an integral component of any video game and can significantly impact user experience. Due to the availability of various unobtrusive motion sensing devices (e.g., Microsoft Kinect, Leap Motion, Creative Interactive Gesture Camera), many gesture based menu systems [4, 7, $10,24]$ have been explored both in academia and commercially in recent years. However, these menu selection methods are often slow (taking about 3-5 seconds) to perform and can suffer from accuracy problems making them less desirable compared to traditional keyboard-mouse or button based menu systems. Since response time and ease of use of a menu system can significantly affect user experience in applications (such as video games), it is essential that they be fast, efficient, and not be a burden on the user while setting up and during play.

People often use fingers to count or enumerate a list of items. In the past, such finger-counting strategies have been investigated for interaction with multi-touch surfaces [3] and distant displays [4]. However, a gestural input system based on finger count gestures (e.g., holding up two fingers) also holds the potential to be a natural and intuitive approach for menu selection in gesture- and motion-based games (see Figure 1). We posit that using one's fingers for menu selection offers several distinct advantages. First, finger count gestures are easily understood (assuming appropriate menu design) and are fast to perform. Second, users do not need to move the cursor to different locations on the screen since finger count gestures are not dependent on the layout of menu items. Third, since no 
cursor movement is needed with finger count menus, possible errors in menu item selection with motion controlled devices are also minimized.

Based on these suppositions, we explored the utility of finger count gestures in two user evaluations. First, we compared a finger count based menu selection approach (Finger-Count menu) against two other gestural menu selection techniques (Hand-n-Hold and Thumbs-Up menu) adapted from existing motion controlled video games. We examined both menu depth and different menu layouts. Second, we compared the Finger-Count menu with 3D marking menus (adapted from Marking menus proposed by Zhao et al. [26] ). In this evaluation, both menu selection strategies also had an expert selection mode (where users can select menu items without the menu appearing on screen). In both experiments, we examined selection time, accuracy, and user preference.

\section{RELATED WORK}

As mentioned in the introduction, menu systems based on finger counting are not a new technique. Finger-Count menus were first proposed for multi-touch surfaces [3]. They were later adapted for distant displays [4], using the Kinect as the gestural input device. However, the algorithm presented by [4] is too slow (average selection time of 8.5 seconds) to be used in realistic scenarios. Additionally, the technique was limited to a circular layout and required both hands \& multiple gestures to select an item. We have improved the selection time of this technique by simplifying it to use a single gesture for selection and by using a faster finger recognition algorithm (adapted from the Intel Perceptual Computing SDK), resulting in an average selection time that is more suitable for our user evaluations and practical use.

Vision-based hand and finger recognition algorithms have been explored by many researchers. The Kinect is a popular choice as input device for some of these algorithms [17, 22, 23]. Jennings et al. [13] used multiple cameras for finger tracking. Kölsch et al. [15] proposed a robust hand detection algorithm based on a single camera but their technique requires a classifier to be trained prior to gesture recognition. Trigo et al. [25] proposed an algorithm for detecting finger tips based on template matching. All these techniques are mostly focused on algorithm design and not on investigating interesting interaction mechanisms based on finger gestures.

Marking menus proposed by Kurtenbach [18] are gesture based menus where the menu items are arranged in a circle and selection is performed by drawing a mark from the center of the menu towards the desired item. Marking menus support two modes: novice and expert. In novice mode, the user selects a menu item from a circular menu displayed on a screen. In expert mode, the menu is not displayed, forcing a user to trace a continuous sequence of marks from memory, which is then recognized by the system. FlowMenus by Guimbretiere and Winograd [11] are also based on the Marking menu. FlowMenus let users make a sequence of selections without moving the pen away from the touch surface but no user evaluations were done as part of this work. Zhao et al. [26] proposed multi-stroke Marking menus with improved accuracy where a user performs a sequence of simple marks instead of a single complex trail. Recently, Marking menus have also been adapted for menu selection in 3D gestural environments [21]. We based our implementation of 3D Marking menus on multi-stroke Marking menus [26] because of its higher selection accuracy.

Researchers have also explored selection performance of several layouts for menu items on screen. Callahan et al. [6] showed that menu items in a circular layout can reduce selection time compared to a linear layout in a 2D plane. A similar result was obtained by Komerska and Ware [16] for their haptic menu system designed for Fishtank VR. Chertoff et al. [7] designed a Nintendo Wiimote based menu system and found pie menus to be faster than linear lists. The results of all these studies are in line with Fitts's law [9], as pie layouts provide a smaller average distance to menu items.

Several menu techniques have been proposed for virtual environments. TULIP [5] menus assign a menu item to each finger of a pinch glove and selection is made by pinching a finger with the thumb. Ni et al. [24] developed the rapMenu which is based on hand gestures and requires a pinch glove. To select an item using the rapMenu, the user rotates his wrist to highlight a group of four menu items and then a finger is pinched with the thumb. Spin menus [10] arrange items on a portion of a circle and enabled selection by rotating the wrist in a horizontal plane. Their system used a workbench from BARCO and an Intersense IS900 Tracker with a wand as an interaction device. Ring menus [19] also arrange items in a circle and attached a tracking device to the user's hand. To select an item, users would rotate their hand and move the desired item into a selection bucket. Body centered menus [20] assign menu items to parts of a user's body (head not included). These menus do not support hierarchical menu items and due to limited mapping locations on body, the number of menu items is also limited. All these techniques use expensive hardware and are not feasible options for consumer oriented products. For our experiments, we used the Creative Interactive Gesture Camera (readily available for \$150) for gesture input. However, it should be noted that the techniques examined in this paper can be implemented using any input device that can recognize hands and fingers. To best of our knowledge, this work is the first to systematically explore the usefulness of finger-based menu selection, especially with relevance to menu item layout and hierarchy, given prior Finger-Count menu selection approaches were not fast enough for thorough user testing.

\section{MENU SELECTION TECHNIQUES}

This section describes the Hand-n-Hold menu, Thumbs-Up menu, Finger-Count menu, and 3D Marking menu. All these techniques were implemented using a finger/hand recognition algorithm adapted from the fingertip/hand detection algorithm included in the Intel's Perceptual Computing SDK [1]. The main properties of these menu techniques are summarized in Table 1. The Creative Interactive Gesture Camera operates at an input frequency of 30 frames per second. We delineate the beginning and end of a selection event by utilizing a frame window of 15 frames to help with segmentation. Thus, each technique requires the user to maintain the selection pose for 0.5 seconds. 


\begin{tabular}{|l|c|c|c|c|}
\hline & Hand-n-Hold & Thumbs-Up & Finger-Count & 3D Marking \\
\hline Gestures & Static & Dynamic & Static & Dynamic \\
\hline Cursor Movement Required? & Yes & Yes & No & Yes \\
\hline Expert Mode Supported? & No & No & Yes & Yes \\
\hline Selection time dependent on Layout? & Yes & Yes & No & Yes \\
\hline
\end{tabular}

Table 1. Properties of menu techniques

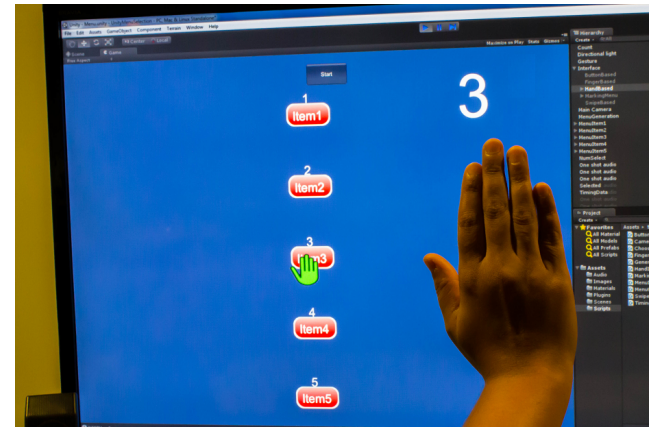

Figure 2. Hand-n-Hold menu with vertical layout.

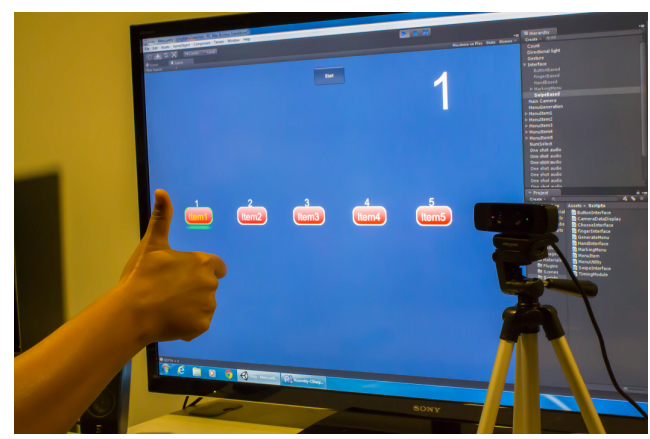

Figure 3. Thumbs-Up menu with horizontal layout.

\section{Hand-n-Hold Menu}

In this technique, users control a cursor by moving their hand in the air (see Figure 2). The position of the cursor on screen is directly related to the $2 \mathrm{D}$ position of their hand in a virtual plane. A menu item is selected by holding the cursor over the desired item for a short duration (about one second). If the menu item has a sub-menu then the sub-menu appears in place (replacing the current menu items). The sub-menu items are selected in the same manner as the main menu. This technique requires visual feedback and supports any layout (horizontal, vertical, and circular were implemented) of items. As a pointer based technique, the efficiency of this menu technique is dependent in part on how the items are arranged on screen.

\section{Thumbs-Up Menu}

A user holds her fist in front of the input device (see Figure 3). The user then has to move her fist either horizontally, vertically or radially in a virtual plane, depending on the layout, to highlight an item corresponding to their fist position and then give a thumbs up gesture to confirm the selection. Sub-menus appear in place and the selection strategy is the same for submenus. Visual feedback is also required for this technique.

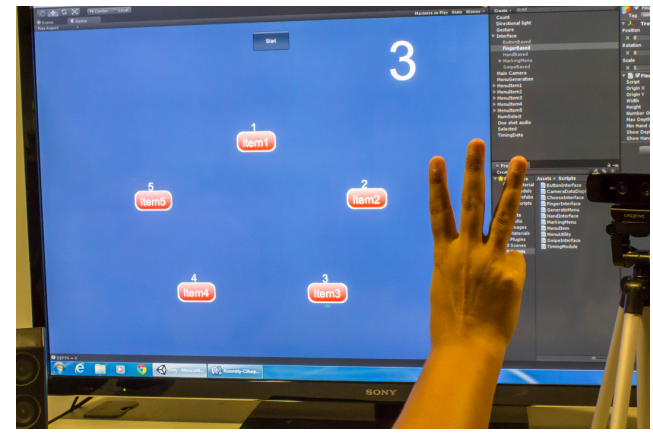

Figure 4. Finger-Count menu with circular layout.

We chose to use the fist for pointing at menu items because it is extremely easy to transition into the Thumbs-Up gesture from the pointing stance. This technique is similar to Hand-nHold in that both require the user to point to an item and then confirm the selection. Hand-n-Hold implements an implicit confirmation mechanism based on a timeout while ThumbsUp requires explicit confirmation from a user. Like Hand-nHold, this technique is layout dependent, and consequently, its efficiency also depends in part on the spatial arrangement of menu items. It is important to note that while we chose to use a fist for pointing at menu items, theoretically, any hand posture can be used for this purpose, followed by any other gesture for confirmation.

\section{Finger-Count Menu}

All the menu items are numbered and the user has to extend a corresponding number of fingers to select a given item (see Figure 4). Items can be arranged in any layout and sub-menus appear in place. We tested three different layouts: horizontal, vertical and circular for this technique. Eyes-free selection is supported since visual feedback is not needed as long as the user knows the corresponding number of the desired item. In novice mode, the menu appears on screen with a number displayed next to each item and the user has to extend a corresponding number of fingers to select an item. In expert mode, the menu does not appear but the selection strategy is the same as novice mode. Expert mode supports a series of finger gestures (extending the appropriate number of fingers) to get to an item under a sub-menu.

This techniques supports using both hands simultaneously, so we can have up to 10 items on screen at a time. In case there are more items, we can label the last item as "Next" indicating that there are more items. If the user selects "Next" then more items appear on screen in place of the original menu. We can extend this idea to include any number of items. Similarly, 
the last item under a sub-menu can be labeled as "Back." The user can select "Back" to reduce the menu depth and see the parent menu in place.

\section{D Marking Menu}

Our 3D Marking menu design is based on the multistroke Marking menu [26] because of its higher selection accuracy. The 3D Marking menu gestures are easy to learn and menu item locations can be remembered easily due to spatial memory [3]. In this technique, the user performs a series of simple gestures instead of a compound stroke. Menu items are always presented to the user in a circular layout. To select an item, the user positions her fist in the center of the menu and moves it towards the desired item, followed by a thumbs up gesture to finalize the selection. Sub-menus appear in place and the selection strategy is the same as the main menu. In novice mode, the menu appears on screen and a single selection is made at a time. In expert mode, the menu is not shown and the user has to perform the required gestures to select an item from memory.

\section{USER EVALUATIONS}

We conducted two experiments to evaluate the usefulness of Finger-Count menus. Our first experiment focused on comparing Finger-Count menus with Hand-n-Hold and ThumbsUp menu selection techniques. We also conducted a second experiment to compare Finger-Count menus with 3D Marking menus. We chose to conduct two experiments because 3D Marking menus support only circular layouts and were very different from Hand-n-Hold and Thumbs-Up. In our pilot tests with two participants, we found the Finger-Count menu to be the fastest technique, therefore we chose to compare only Finger-Count menus with 3D Marking menus. We chose a within-subjects design for our experiments in order to be able to measure and compare user perceptions of the menu selection techniques on a variety of quantitative and qualitative metrics. All menu items were labeled with numbers in our experiments. The setup and participants were the same for both experiments. Participants completed both experiments in order (experiment 1 followed by experiment 2) in a single session. We had the following hypotheses about the chosen menu selection techniques:

Hypothesis 1 (H1) : Finger-Count menus are faster than the other menu techniques.

Hypothesis 2 (H2) : Finger-Count menus have higher selection accuracy than the other menu techniques.

Hypothesis 3 (H3) : People will prefer to use Finger-Count Menus than the other techniques.

\section{Subjects and Apparatus}

We recruited 36 participants (31 males and 5 females ranging in age from 18 to 33) from the University of Central Florida, of which two were left handed. The experiment duration ranged from 50 to 70 minutes and all participants were paid $\$ 10$ for their time.

The experiment setup, shown in Figure 5, consisted of a 55" Sony HDTV and the Creative Interactive Gesture Camera (a readily available and affordable depth sensing camera)

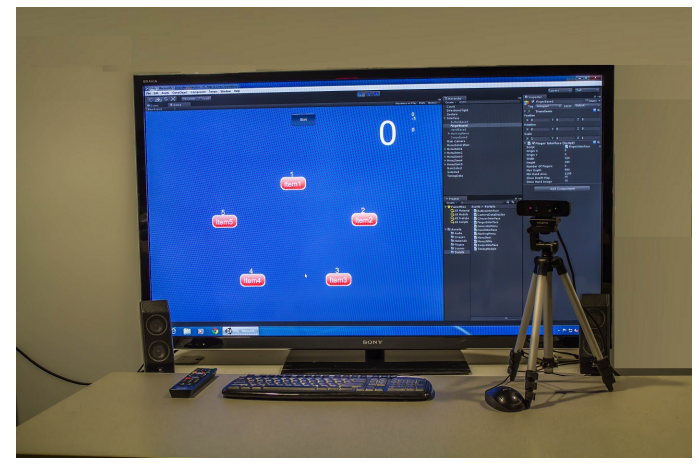

Figure 5. The Experimental Setup.

mounted on a mini tripod. We used the Unity3D game engine [2] and Intel Perceptual Computing Software Development Kit (PCSDK) [1] for implementing all four menu techniques. Participants were seated about 3 feet away from the display and the camera was placed about 1.5 feet away from the participant, in order to ensure that the participant's hand was completely visible to the camera. The position of the camera was changed either to the left or right of the participant, while maintaining the distance from the participant, based on dexterity (left handed or right handed) in order to enable optimal viewing of the menu items on screen.

\section{Procedure}

The experiment began with the participant seated in front of the TV and the moderator seated to the side. Participants were given a consent form that explained the experiment procedure. They were then given a pre-questionnaire which collected general information about the participant (age, sex, dexterity, etc.). Participants then completed both experiments in order. At the beginning of each experiment, the moderator explained the selection techniques and allowed the user to practice each technique for as long as necessary. Details of experiment tasks are provided in the respective sub-sections of the experiments.

We recorded selection time and accuracy of all the techniques presented in both experiments. For both experiments, selection time was measured as the time from when a random number appeared on screen to the time the corresponding item was selected. Selection accuracy of a technique was measured as the percentage of correct selections out of total selections made for that technique. After each experiment, the participant filled out a post-questionnaire (see Table 2) with questions about their experiences with the techniques they tried.

\section{Experiment 1: Hand-n-Hold, Thumbs-Up, and Finger- Count Menu Comparison}

The first experiment compared Hand-n-Hold, Thumbs-Up, and Finger-Count menus. All these techniques support horizontal, vertical and circular layouts. Hand-n-Hold and Thumbs-Up only support single handed interactions. As a result, we chose to use a one handed variation of the FingerCount menu in order to remove a potential confounding variable. Moreover, Hand-n-Hold menu and Thumbs-Up menu 
Table 2. Post-Questionnaire. Participants responded to question 1-8 on a 7 point Likert scale. Question 9 was a multiple choice question.

\begin{tabular}{|l|l|}
\hline \multicolumn{2}{|c|}{ Post Experiment Questions } \\
\hline Q1 & To what extent did you like this menu selection technique? \\
\hline Q2 & How mentally demanding was this technique? \\
\hline Q3 & To what extent your arm was tired when using this technique? \\
\hline Q4 & Did you feel hurried or rushed when using this technique? \\
\hline Q5 & $\begin{array}{l}\text { How successfully you were able to choose the items you were } \\
\text { asked to select? }\end{array}$ \\
\hline Q6 & Did you feel that you were trying your best? \\
\hline Q7 & To what extent you felt frustrated using this technique? \\
\hline Q8 & $\begin{array}{l}\text { To what extent did you feel that this technique was hard to } \\
\text { use? }\end{array}$ \\
\hline Q9 & $\begin{array}{l}\text { Which layout of menu items would you prefer for this tech- } \\
\text { nique? Horizontal, vertical, circular or all equally? }\end{array}$ \\
\hline
\end{tabular}

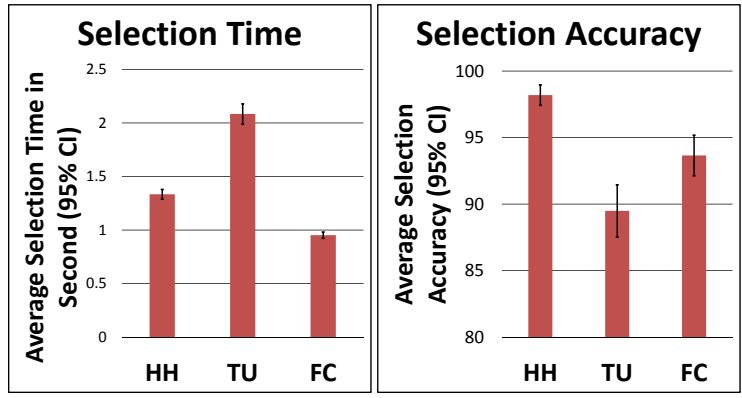

Figure 6. Average selection time and accuracy of each technique where HH is Hand-n-Hold, TU is Thumbs-Up and FC is Finger-Count menu.

do not support expert mode so we did not have any expert mode as part of this experiment.

\section{Experiment Design}

This within-subjects experiment had 3 independent variables: technique (Hand-n-Hold, Thumbs-Up, and Finger-Count), layout (horizontal, vertical and circular) and menu depth (0 and 1 ). In total we had $3 \times 3 \times 2=18$ conditions and for each condition the user conducted 10 trials which makes a total of 180 selections per participant as part of this experiment. Our dependent variables were average menu selection time and selection accuracy, where the average is taken over the 10 trials for that condition.

Each condition was presented to the user in random order based on a Latin square design [8]. For each condition, users were asked to select 10 randomly generated items displayed on screen one item at a time. After completing the experiment, users filled a post-questionnaire (see Table 2) with the same set of questions for each technique and then ranked the techniques based on ease of use, arm fatigue, efficiency, and overall best.

\section{Quantitative Results}

We used repeated-measures 3-factor ANOVA per dependent variable. We did a post-hoc analysis using pairwise sample t-tests. We used Holm's sequential Bonferroni adjustment to correct for type I errors [12] and the Shapiro-Wilk test to make sure the data was parametric.

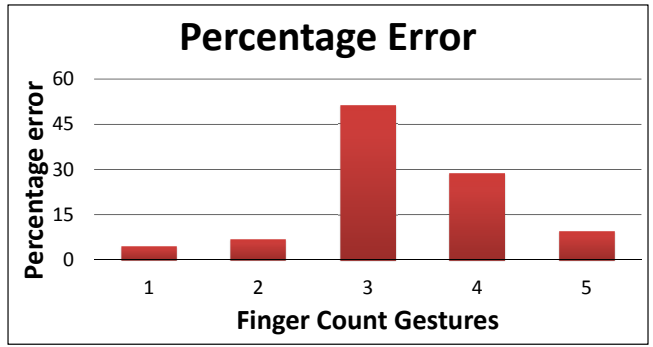

Figure 7. Error percentage (out of $6.81 \%$ errors) of individual gestures for Finger-Count menu. Most of the errors were due gesture 3.

Mean selection time and selection accuracy for each technique is shown in Figure 6. We found significant differences in mean selection time $\left(F_{2,34}=363.657, p<0.005\right)$ and selection accuracy $\left(F_{2,34}=45.758, p<0.005\right)$ between the menu techniques. The Finger-Count menu was faster than Hand-n-Hold $\left(t_{35}=-21.505, p<0.005\right)$ and Hand-n-Hold was faster than Thumbs-Up $\left(t_{35}=-21.433, p<0.005\right)$. Hand-n-Hold was more accurate than the Finger-Count menu $\left(t_{35}=-5.586, p<0.005\right)$, which in turn was more accurate than Thumbs-Up $\left(t_{35}=4.488, p<0.005\right)$.

The Finger-Count menu was the only technique that uses different gestures (different number of fingers extended) for different numbered items. Therefore, we also analyzed the individual gesture error percentage (see Figure 7) and found an overall error rate of $6.81 \%$, with $51.09 \%$ of the errors attributed to the gesture for number 3 (three fingers extended) and $28.46 \%$ due to the gesture for number 4 (four finger extended).

Menu depth did not have any significant effect on selection time $\left(F_{1,35}=1.340, p=0.255\right)$. Depth showed significant effect on accuracy $\left(F_{1,35}=0.258, p<0.05\right)$ but post-hoc analysis did not find any significant differences.

We also found that the layout of menu items significantly affects the mean selection time of all techniques $\left(F_{2,34}=\right.$ 9.384, $p<0.005)$. However, there was no significant effect of item layout on mean selection accuracy $\left(F_{2,34}=\right.$ $2.651, p=0.135)$. Horizontal layouts were faster than vertical layouts $\left(t_{35}=-3.095, p<0.005\right)$ and circular layouts $\left(t_{35}=-4.243, p<0.005\right)$. There was no significant difference in average selection time between vertical layout and circular layout.

We also analyzed each technique separately to study the effects of layout (see Figure 8 and 9). The results are as follows:

Hand-n-Hold Menu Layout had significant effect only on accuracy $\left(F_{2,34}=5.548, p<0.05\right)$. A post-hoc analysis revealed that the circular layout was significantly more accurate than the horizontal layout $\left(t_{35}=-3.366, p<\right.$ $0.005)$.

Thumbs-Up Menu Layout had significant effect on time $\left(F_{2,34}=20.563, p<0.005\right)$ and accuracy $\left(F_{2,34}=\right.$ $7.776, p<0.005)$. The horizontal layout was significantly faster than the vertical $\left(t_{35}=-4.075, p<0.005\right)$ and the 


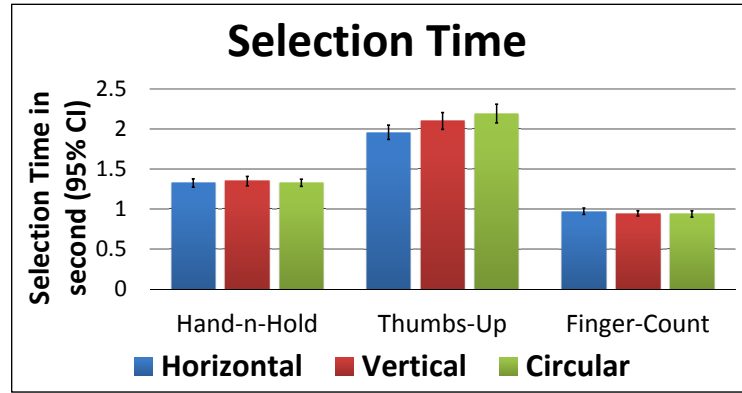

Figure 8. Average selection time of each menu technique for different layouts. The Hand-n-Hold and Finger-Count menus did not have any significant changes in selection time with layout.

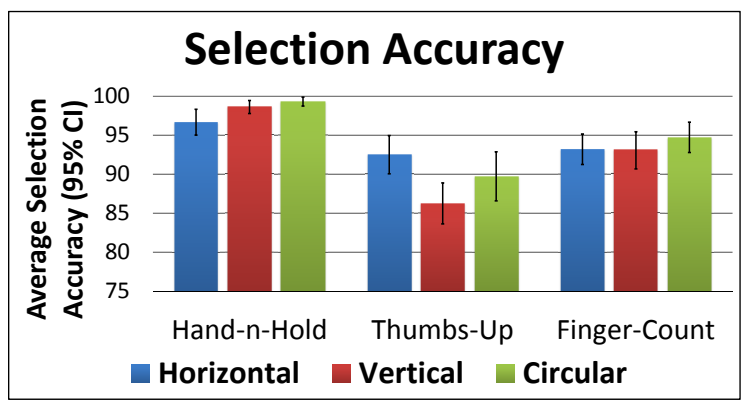

Figure 9. Average selection accuracy for each layout.

circular layout $\left(t_{35}=-5.831, p<0.005\right)$. The horizontal layout was significantly more accurate than the vertical layout $\left(t_{35}=3.668, p<0.005\right)$.

Finger-Count Menu As expected, layout had no effect on selection time and accuracy of the Finger-Count menu.

\section{Qualitative Results}

Based on the post-questionnaire data, 22 people preferred the circular layout for the Hand-n-Hold menu, 21 preferred the horizontal layout for the Thumbs-Up menu, and 32 people thought that all layouts were equivalent for the Finger-Count menu (see Figure 10). The Finger-Count menu was ranked as the overall best technique and the Thumbs-Up menu as the worst technique. The Finger-Count menu was also ranked as best (see Figure 11) in terms of ease of use, efficiency and arm fatigue (less fatigue is better).

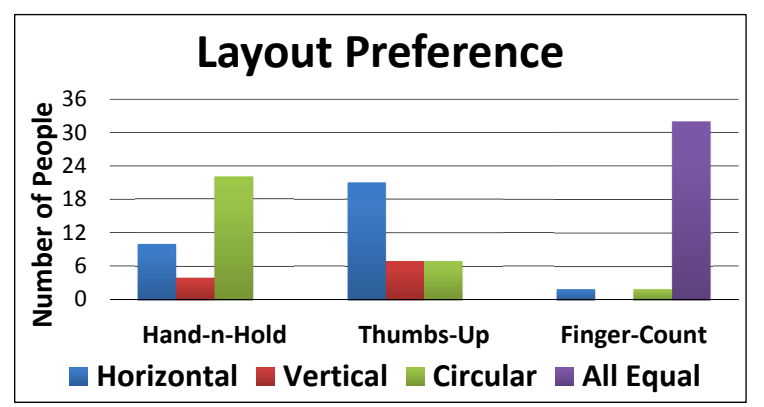

Figure 10. Layout preference for each technique. Finger-Count menus are layout independent.

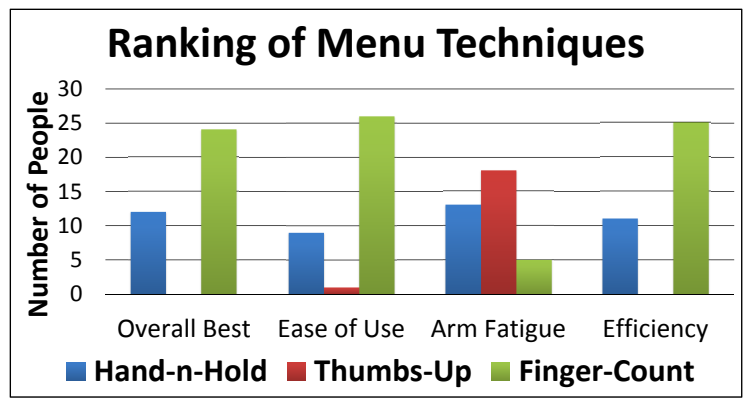

Figure 11. Ranking of techniques based on overall best, ease of use, arm fatigue, and efficiency. Finger-Count menu was ranked as the best technique by majority of participants.

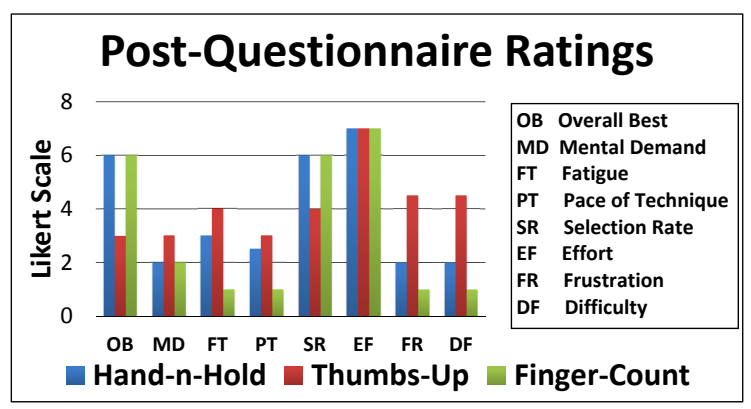

Figure 12. Median ratings for post-questionnaire questions for each technique.

To analyze the Likert scale data, we used Friedman's test and then a post-hoc analysis was done using Wilcoxon signed rank tests. These results are displayed in Table 3. Median rating for post-questionnaire questions 1 to 8 is summarized in Figure 12. From the results we an see that:

- People liked Hand-n-Hold and Finger-Count more compared to Thumbs-Up.

- Finger-Count and Hand-n-Hold are mentally less demanding than Thumbs-Up.

- Finger-Count causes less arm fatigue compared to Hand-nHold and Thumbs-Up.

- For Thumbs-Up, more people thought they were not able to select items they were asked to select than Hand-n-Hold and Finger-Count.

- Frustration level was higher for Thumbs-Up than Hand-nHold and Finger-Count.

- People thought that Thumbs-Up was significantly harder to use than Hand-n-Hold and Finger-Count.

\section{Experiment 2: Compare Finger-Count Menu with 3D Mark- ing Menu}

This experiment focused on comparing the Finger-Count menu with a 3D Marking menu. 3D Marking menus support only a circular layout, so we restricted the Finger-Count menu to a circular layout for a fair comparison. Menu depth for this experiment was set to one in order to the keep the same environment for both novice and expert mode. As 3D Marking 


\begin{tabular}{|c|c|c|c|c|}
\hline Question & Friedman's test & HH vs TU & HH vs FC & TU vs FC \\
\hline Q1 & $\chi^{2}(2)=41.603, p<0.0005$ & $Z=-4.389, p<0.005$ & $Z=-1.649, p=0.099$ & $Z=-4.907, p<0.005$ \\
\hline Q2 & $\chi^{2}(2)=19.855, p<0.0005$ & $Z=-3.809, p<0.005$ & $Z=-1.029, p=0.304$ & $Z=-3.151, p<0.005$ \\
\hline Q3 & $\chi^{2}(2)=35.138, p<0.0005$ & $Z=-1.524, p=0.128$ & $Z=-3.780, p<0.005$ & $Z=-4.386, p<0.005$ \\
\hline Q4 & $\chi^{2}(2)=17.196, p<0.0005$ & $Z=-2.656, p<0.010$ & $Z=-1.837, p=0.066$ & $Z=-3.197, p<0.005$ \\
\hline Q5 & $\chi^{2}(2)=35.613, p<0.0005$ & $Z=-4.459, p<0.005$ & $Z=-0.996, p=0.334$ & $Z=-3.972, p<0.005$ \\
\hline Q6 & $\chi^{2}(2)=3.250, p=0.197$ & $Z=-0.000, p=1.000$ & $Z=-1.076, p=0.282$ & $Z=-0.964, p=0.335$ \\
\hline Q7 & $\chi^{2}(2)=41.407, p<0.0005$ & $Z=-4.778, p<0.005$ & $Z=-0.574, p<=0.566$ & $Z=-4.330, p<0.005$ \\
\hline Q8 & $\chi^{2}(2)=41.333, p<0.0005$ & $Z=-4.890, p<0.005$ & $Z=-0.330, p=0.742$ & $Z=-4.523, p<0.005$ \\
\hline
\end{tabular}

Table 3. Results of Friedman's test and post-hoc analysis for Likert scale data of Experiment 1. (HH : Hand-n-Hold, TU: Thumbs-Up and FC: Finger-Count)

menus also only support interaction using a single hand, we again restricted the Finger-Count menu to use a single hand, resulting in a maximum of 5 items per menu.

\section{Experimental Design}

Our second within-subjects experiment had two independent variables: technique (Finger-Count and 3D Marking menu) and user mode (novice and expert). There were a total of $2 \times 2=4$ conditions with 10 trials for each making it a total of 40 selections per participant. Our dependent variables were average menu selection time and average selection accuracy, where the average is taken over 10 trials for that condition. Each condition was presented to the user in a random order based on a Latin square design [8]. In novice mode, users were asked to select 10 randomly generated items. In expert mode, a sequence of two numbers were generated for each trial and users were asked to pick the corresponding items in order. After completing the experiment, users filled out a post-questionnaire (only questions 1 to 8 of Table 2 ) with the same set of questions for each technique.

\section{Quantitative Results}

A repeated-measures 2-factor ANOVA was used per dependent variable. We did a post-hoc analysis using pairwise sample t-tests. We used Holm's sequential Bonferroni adjustment to correct for type I errors [12] and the Shapiro-Wilk test to make sure the data was parametric. Table 4 shows the results of repeated measures two-factor ANOVA analysis. The Finger-Count menu was significantly faster (see Figure 13) than the 3D Marking menu in both novice mode $\left(t_{35}=\right.$ $11.868, p<0.0005)$ and expert mode $\left(t_{35}=10.942, p<\right.$ $0.0005)$. In novice mode, the average selection time was 0.933 seconds $(\sigma=0.098)$ for the Finger-Count menu and 2.09 seconds ( $\sigma=0.643)$ for the 3D Marking menu. In expert mode, selection time was 2.307 seconds $(\sigma=0.223)$ for the Finger-Count menu and 4.024 seconds $(\sigma=1.067)$ for the 3D Marking menu. Overall, there was no significant difference in selection accuracy between the menu techniques (see Figure 13). Novice mode had significantly higher selection accuracy than expert mode $\left(t_{35}=3.448, p<0.005\right)$. Average selection accuracy was $96.25 \%(\sigma=5.123)$ for novice mode and $91.25 \%(\sigma=9.131)$ for expert mode.

\section{Qualitative Results}

The qualitative data was analyzed separately for novice and expert modes. To analyze the Likert scale data, we used Wilcoxon signed rank tests. Median ratings for postquestionnaire questions 1 to 8 are summarized in Figure 14.

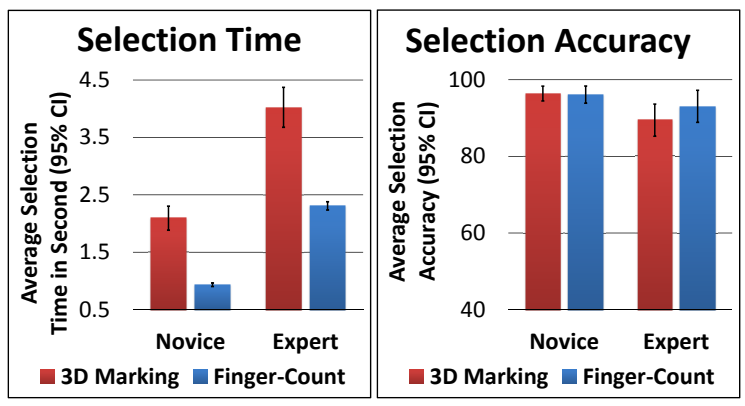

Figure 13. Selection time and accuracy by technique and mode.

In novice mode (see Figure 14), people liked the FingerCount menu significantly more than the 3D Marking menu $(Z=-4.059, p<0.0005)$. The 3D Marking menu is significantly more mentally demanding than the Finger-Count menu $(Z=-3.272, p<0.005)$. The 3D Marking menu also lead to significantly more arm fatigue than the FingerCount menu $(Z=-3.383, p<0.005)$. People thought that using the Finger-Count menu let them select items with significantly higher accuracy than the 3D Marking menu $(Z=-3.106, p<0.005)$. People also felt significantly less frustrated with the Finger-Count menu than the 3D Marking menu $(Z=-3.778, p<0.0005)$. Finally, the 3D Marking menu was significantly harder to use than the Finger-Count menu $(Z=-3.357, p<0.005)$.

Statistics for expert mode were similar to novice mode (see Figure 14). In expert mode, people liked the Finger-Count menu significantly more than the 3D Marking menu $(Z=$ $-4.335, p<0.0005)$. The 3D Marking menu is significantly more mentally demanding than the Finger-Count menu $(Z=-4.196, p<0.005)$. 3D Marking menu usage also lead to significantly more arm fatigue than the FingerCount menu $(Z=-4.115, p<0.0005)$. People thought that when using Finger-Count menus they were able to select items with significantly higher accuracy than with the 3D Marking menu $(Z=-3.751, p<0.005)$. People felt significantly less frustrated with the Finger-Count menu $(Z=-3.348, p<0.005)$. Finally, the 3D Marking menu was significantly harder to use than the Finger-Count menu $(Z=-4.307, p<0.0005)$.

\section{DISCUSSION}

Our experiments indicate that Finger-Count menus let participants select items significantly faster than either Hand-n- 


\begin{tabular}{|c|c|c|}
\hline Source & Selection Time & Accuracy \\
\hline Technique & $F_{1,35}=145.774, p<0.0005$ & $F_{1,35}=0.864, p=0.359$ \\
\hline Mode & $F_{1,35}=751.146, p<0.0005$ & $F_{1,35}=11.887, p<0.005$ \\
\hline Technique $\times$ Mode & $F_{1,35}=26.831, p<0.0005$ & $F_{1,35}=1.755, p=0.194$ \\
\hline
\end{tabular}

Table 4. Repeated measure 2-factor ANOVA analysis for comparing the 3D Marking menu with the Finger-Count menu. There was significant difference in selection time based on menu technique as well as mode. Accuracy was significantly different between the user modes.

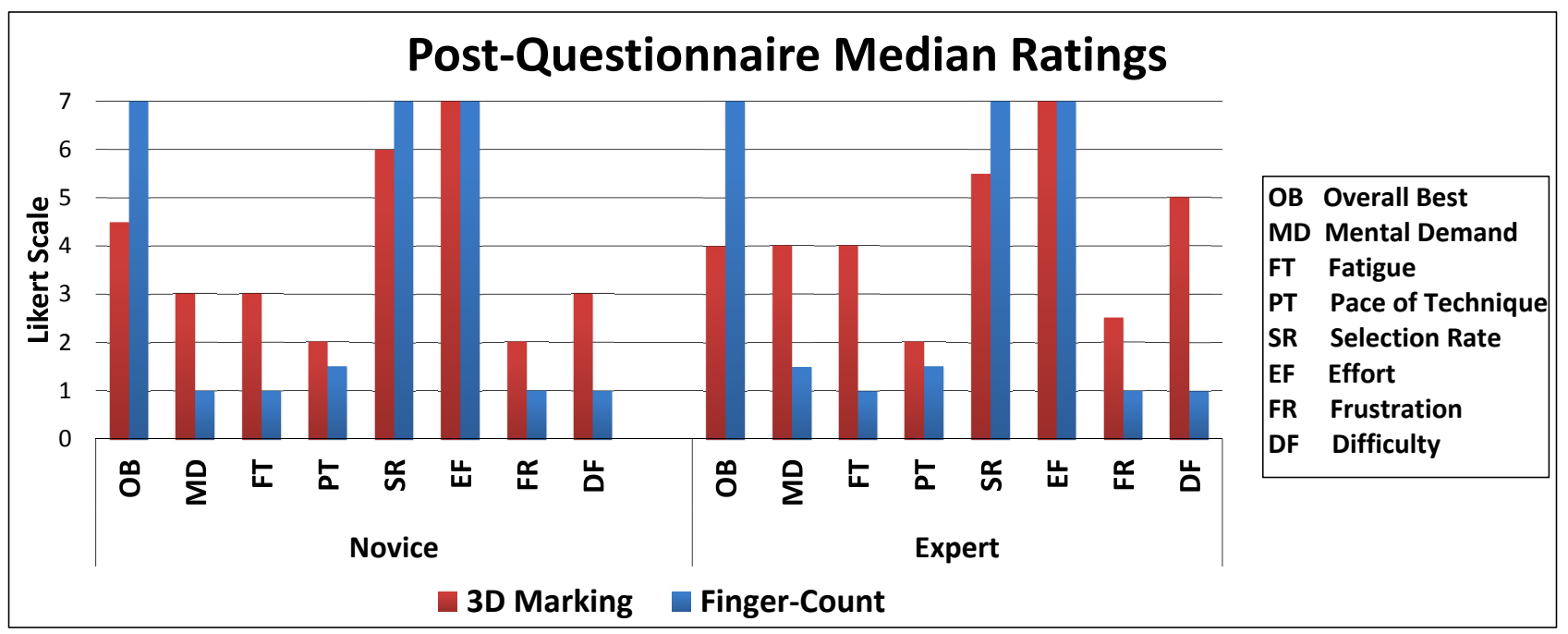

Figure 14. Median ratings for post-questionnaire questions for each technique.

Hold, Thumbs-Up or 3D Marking menus. This is primarily because Finger-Count menus do not require the user to move their hand in accordance with the position of items on screen, resulting in a constant selection time for all items. For Handn-Hold menus, the second-fastest technique, the user has to continuously move his hand to select an item, increasing the selection time. Thumbs-Up not only requires a user to move his hand for selecting a menu item, but to also give a thumb's up gesture to finalize the selection. 3D Marking menus have similar hand motion characteristics as Thumbs-Up, as both techniques require hand motion and then an explicit thumbsup gesture to finalize item selection. This additional motion and a gesture takes significantly more time than simply extending one's fingers.

We found that Hand-n-Hold was the most accurate out of all selection techniques tested because it involves controlling a pointer with one's hand with implicit finalization, making it less error prone than recognizing extended fingers or the thumb's up gesture. Even though Hand-n-Hold is the most accurate technique, we found that users preferred Finger-Count menus more because of its faster selection time and its natural interaction metaphor. In the future, we foresee better selection accuracy for Finger-Count menus due to the availability of better gestural input devices and recognition algorithms.

Our analysis of menu item layout presents an interesting picture. Finger-Count menus have a constant selection time and are not at all affected by the layout of menu items. For Handn-Hold, item layout did not have any effect on selection time but circular layouts resulted in higher selection accuracy than horizontal and vertical layouts, probably due to the similar spacing of menu items, resulting in a similar amount of movement. When using Hand-n-Hold with horizontal and vertical layouts, participants occasionally tended to accidentally bump into wrong items while moving the pointer to a desired item, resulting in a wrong selection. But with circular layouts, they could keep the pointer inside the circle and reach all menu items at the periphery without accidentally selecting other items. For Thumbs-Up, we found that horizontal layouts resulted in faster selection and increased selection accuracy. We believe this is primarily because a person's arm has a more natural and relaxed posture when moved horizontally. In the case of vertical and circular layouts, participants often oriented their hand in such a way that their thumb was not pointing upwards making it difficult for the gesture recognizer to identify it as a thumb's up gesture. This orientation decreased the mean selection accuracy in these layouts for Thumbs-Up.

For Finger-Count menus, three fingers can present a possibly difficult combination for detection because users in our experiments tended to keep the middle finger and ring finger close enough to be detected as a single finger. However, detecting the number four proved easier because participants automatically provided sufficient spacing to alleviate confusion in the recognizer. This issue can also be remedied by using both hands simultaneously (e.g., index finger in one hand and index $\&$ middle finger in the other hand to indicate a 3 gesture). Our implementation of Finger-Count menus support using both hands simultaneously. But since the other techniques in our study were single handed only, we restricted Finger-Count menus to use single hand interaction for a fair comparison. 
Our subjective responses indicate that Finger-Count menus were the most preferred and most efficient, had the least arm fatigue, and was the least frustrating technique. This seems promising for future games and applications with short range gestural input. Participants were impressed by the selection time of the Finger-Count menus. The second most preferred technique was Hand-n-Hold because of its ease of use and high accuracy. People are used to controlling a pointer using a mouse and this technique seems familiar to them. People did not like Thumbs-Up because of high error rate. Participants thought that 3D Marking menus are more mentally demanding than the Finger-Count menu. This is because for Finger-Count menus, the user does not have to worry about the location of items on screen. This fact is much more noticeable in expert mode where the menu does not appear on screen. For 3D Marking menus, people need to memorize the location of items with respect to the center to be able to perform a radial mark to select the desired item. Finger-Count menus were rated as less frustrating and most liked technique than the 3D Marking menu.

Based on the results of our experiments, we were able to accept H1, H3 and were unable to accept H2. Consequently, we believe that Finger-Count menus have the potential to be used as a menu system in future 3D gesture controlled applications and video games. Finger-Count menus have a very low response time making users spend a minimal amount of time interacting with menus. All the Finger-Count gestures are intuitive and easy to remember. Both casual and expert gamers could use this technique with a limited learning curve. Once players get used to the menu system, they can transition to expert mode and can change game setting (e.g., change appearance of game character, selecting a weapon from inventory, switching camera position in racing games, etc.) with no interference from menu items on screen. It could also be a good idea to mix traditional mouse based menus with Finger-Count menus. Mouse pointer menus could be used to select game settings at the beginning of a game and FingerCount menus for changing in-game settings while playing. For example, in a gesture controlled car racing game, a user can set display resolution, select a track and car using traditional menus. While racing, he can switch between first person view to third person view using Finger-Count menus. Similarly, a user could select weapons from an inventory for a First Person Shooter (FPS) games using Finger-Count menus. Finger-count menus could be combined with other hand gestures to increase the number of possible gestures thereby increasing the number of possible motion controlled tasks in video games.

There are a few factors that could have affected our results. When comparing layouts for a given technique, items were equally spaced for a given layout but the item spacing was not the same across the three layouts. It could have a minor effect on our results but we still believe that horizontal layout would be slightly faster than vertical layout for hand based interaction because a person's arm has more natural and relaxed posture when moved horizontally. The shape of the menu items could also have had some influence on how well users perform in an horizontal or vertical layout. Ide- ally, a circular menu item would be more balanced across all dimensions but we don't find such menu items in video games. Hence, circular menu items were not considered to simulate real world menu items. Our study design could also have had an influence on our results. The two experiments were performed in order, experiment 1 and then experiment 2 but conditions in each experiment were randomized. This could have some effect on our results but we believe that people would have still preferred Finger-count menus over 3D Marking menus (in experiment 2) due to its ease of use and fast response time. Moreover, we did not consider studying learning effects because all the gestures performed were easy to learn requiring very little time to train the users.

Finger-Count menus do have some limitations. Hand physiology also plays an important role. Some people found it difficult to keep their fingers separated. One of the participants had arthritis in one hand. It was difficult for him to keep enough separation between the fingers to be counted as separate fingers by the recognizer. But the Finger-Count menu worked fine for him when he used his other hand. We think that it could also be a problematic for some old age people because of the weakening of intrinsic hand muscles with age [14]. Thus, such an interface could be a challenge for people with arthritis or any form of ailment preventing them from keeping their fingers separated for the gesture recognizer.

\section{CONCLUSION AND FUTURE WORK}

We presented an in-depth exploration comparing FingerCount menus with Hand-n-Hold, Thumbs-Up, and 3D Marking menus using different layouts and modes (novice and expert). Our results show that Finger-Count menus are a viable option for 3D menu selection tasks with fast response times and high accuracy and could be well suited for gesture controlled applications such as games. In terms of horizontal, vertical and circular layouts, selection time and selection accuracy of Finger-Count menus did not change with layout. However, the circular layout had higher selection accuracy for Hand-n-Hold menus while the horizontal layout was faster and more accurate for Thumbs-Up menus. A significantly higher number of participants ranked Finger-Count menus as their favorite technique and the second best technique was the Hand-n-Hold menu. In the future, it will be interesting to see how well Finger-Count menus perform in real application environments. We would also like to see the performance of Finger-Count menus in the context of 3D environments where menu item selection is in 3D space.

\section{ACKNOWLEDGMENTS}

This work is supported in part by NSF CAREER award IIS0845921 and NSF awards IIS-0856045 and CCF-1012056. We would also like to thank Salman Cheema \& the other members of ISUE lab for their support and the anonymous reviewers for their useful comments and feedback.

\section{REFERENCES}

1. Intel perceptual computing SDK. http://software.intel.com/en-us/vcsource/tools/ perceptual-computing-sdk.

2. Unity3D. http://unity3d.com/, 2013. 
3. Bailly, G., Lecolinet, E., and Guiard, Y. Finger-count \& radial-stroke shortcuts: 2 techniques for augmenting linear menus on multi-touch surfaces. In Proceedings of the SIGCHI Conference on Human Factors in Computing Systems, ACM (2010), 591-594.

4. Bailly, G., Walter, R., Müller, J., Ning, T., and Lecolinet, E. Comparing free hand menu techniques for distant displays using linear, marking and finger-count menus. In Human-Computer Interaction INTERACT 2011, vol. 6947 of LNCS. Springer Berlin Heidelberg, 2011, 248-262.

5. Bowman, D., and Wingrave, C. Design and evaluation of menu systems for immersive virtual environments. In Virtual Reality, 2001. Proceedings. IEEE (March 2001), $149-156$.

6. Callahan, J., Hopkins, D., Weiser, M., and Shneiderman, B. An empirical comparison of pie vs. linear menus. In Proceedings of the SIGCHI Conference on Human Factors in Computing Systems, CHI '88, ACM (New York, NY, USA, 1988), 95-100.

7. Chertoff, D. B., Byers, R. W., and LaViola, Jr., J. J. An exploration of menu techniques using a 3D game input device. In Proceedings of the 4th International Conference on Foundations of Digital Games, FDG '09, ACM (New York, NY, USA, 2009), 256-262.

8. Fisher, R. A. The design of experiments. Oxford, England: Oliver \& Boyd, 1935.

9. Fitts, P. The information capacity of the human motor system in controlling the amplitude of movement. Journal of Experimental Psychology 47, 6 (Sep 1954), 381-391.

10. Gerber, D., and Bechmann, D. The spin menu: A menu system for virtual environments. In Proceedings of the 2005 IEEE Conference 2005 on Virtual Reality, VR '05, IEEE Computer Society (Washington, DC, USA, 2005), $271-272$.

11. Guimbretiére, F., and Winograd, T. Flowmenu: combining command, text, and data entry. In Proceedings of the 13th annual ACM symposium on User interface software and technology, UIST '00, ACM (New York, NY, USA, 2000), 213-216.

12. Holm, S. A simple sequentially rejective multiple test procedure. Scandinavian Journal of Statistics 6, 2 (1979), 65-70.

13. Jennings, C. Robust finger tracking with multiple cameras. In Recognition, Analysis, and Tracking of Faces and Gestures in Real-Time Systems, 1999. Proceedings. International Workshop on (1999), 152 -160 .

14. Kapur, S., Zatsiorsky, V. M., and Latash, M. L. Age-related changes in the control of finger force vectors. Journal of Applied Physiology 109, 6 (2010), 1827-1841.

15. Kölsch, M., and Turk, M. Robust hand detection. In Proceedings of the Sixth IEEE international conference on Automatic face and gesture recognition, IEEE Computer Society (2004), 614-619.

16. Komerska, R., and Ware, C. A study of haptic linear and pie menus in a $3 \mathrm{~d}$ fish tank vr environment. In Haptic Interfaces for Virtual Environment and Teleoperator Systems, 2004. HAPTICS '04. Proceedings. 12th International Symposium on (March 2004), 224 - 231.

17. Kulshreshth, A., Zorn, C., and LaViola, J. Real-time markerless kinect based finger tracking and hand gesture recognition for HCI. In Proceedings of the IEEE Symposium on 3D User Interfaces (March 2013), 187-188.

18. Kurtenbach, G. The design and evaluation of marking menus. PhD thesis, University of Toronto, Ontario, Canada, 1993.

19. Liang, J., and Green, M. JDCAD: A highly interactive 3D modeling system. Computers \& graphics 18, 4 (1994), 499-506.

20. Mine, M. R., Brooks Jr, F. P., and Sequin, C. H. Moving objects in space: exploiting proprioception in virtual-environment interaction. In SIGGRAPH, ACM (1997), 19-26.

21. Ren, G., and O'Neill, E. 3D marking menu selection with freehand gestures. In IEEE Symposium on $3 D$ User Interfaces (3DUI), IEEE (2012), 61-68.

22. Ren, Z., Yuan, J., and Zhang, Z. Robust hand gesture recognition based on finger-earth mover's distance with a commodity depth camera. In Proceedings of the 19th ACM international conference on Multimedia, MM '11, ACM (New York, NY, USA, 2011), 1093-1096.

23. Ryan, D. J. Finger and gesture recognition with microsoft kinect. Master's thesis, University of Stavanger, Norway, 2012.

24. Tao, N., McMahan, R., and Bowman, D. Tech-note: rapmenu: Remote menu selection using freehand gestural input. In Proceedings of the IEEE Symposium on 3D User Interfaces (March 2008), 55-58.

25. Trigo, T., and Pellegrino, S. An analysis of features for hand-gesture classification. In 17th International Conference on Systems, Signals and Image Processing (IWSSIP 2010) (2010), 412-415.

26. Zhao, S., and Balakrishnan, R. Simple vs. compound mark hierarchical marking menus. In Proceedings of the 17th annual ACM symposium on User interface software and technology, ACM (2004), 33-42. 